\title{
Improving Program Comprehension by Answering Questions (Keynote)
}

\author{
Brad A. Myers \\ Human Computer Interaction Institute \\ School of Computer Science \\ Carnegie Mellon University \\ Pittsburgh, PA, USA \\ bam@cs.cmu.edu \\ www.natprog.org
}

\begin{abstract}
My Natural Programming Project is working on making software development easier to learn, more effective, and less error prone. An important focus over the last few years has been to discover what are the hard-to-answer questions that developers ask while they are trying to comprehend their programs, and then to develop tools to help answer those questions. For example, when studying programmers working on everyday bugs, we found that they continuously ask "Why" and "Why Not" questions as they try to comprehend what happened. We developed the "Whyline" debugging tool, which allows programmers to directly ask these questions of their programs and get a visualization of the answers. In a small lab study, Whyline increased productivity by a factor of about two. We studied professional programmers trying to understand unfamiliar code and identified over 100 questions they identified as hard-to-answer. In particular, we saw that programmers frequently had specific questions about the feasible execution paths, so we developed a new visualization tool to directly present this information. When trying to use unfamiliar APIs, such as the Java SDK and the SAP eSOA APIs, we discovered some common patterns that make programmers up to 10 times slower in finding and understanding how to use the appropriate methods, so we developed new tools to assist them. This talk will provide an overview of our studies and resulting tools that address program comprehension issues.
\end{abstract}

Index Terms-Natural Programming, Debugging, Application Programming Interfaces (APIs), Integrated development environments (IDEs), Documentation, Reverse Engineering, Programming Environments.

\section{SPEAKER SHORT BIOGRAPHY}

Brad A. Myers is a Professor in the Human-Computer Interaction Institute in the School of Computer Science at Carnegie Mellon University. He is an IEEE Fellow, ACM Fellow, and a member of the CHI Academy, an honor bestowed on the principal leaders of the field. He is the principal investigator for the Natural Programming Project and the Pebbles Handheld Computer Project, and previously led the Amulet and Garnet projects. He is the author or editor of over 400 publications, including the books "Creating User Interfaces by Demonstration" and "Languages for Developing User
Interfaces," and he has been on the editorial board of five journals. He has been a consultant on user interface design and implementation to over 75 companies, and regularly teaches courses on user interface design and software. Myers received a $\mathrm{PhD}$ in computer science at the University of Toronto where he developed the Peridot UIMS. He received the MS and BSc degrees from the Massachusetts Institute of Technology during which time he was a research intern at Xerox PARC. From 1980 until 1983 he worked at PERQ Systems Corporation. His research interests include programming environments, programming language design, user interface development systems, user interfaces, handheld computers, programming by example, visual programming, interaction techniques, and window management. He belongs to SIGCHI, ACM, IEEE, and the IEEE Computer Society.

\section{FOR MORE INFORMATION}

The complete list of our publications is provided at our website: www.natprog.org. An (old) overview of the project is [1]. There are many papers about the Whyline [2, 3], the study of hard-to-answer questions [4, 5], the visualization tool to help with reverse engineering [6], some studies of APIs [7-10], and tools to help with APIs [11-15].

\section{ACKNOWLEDGEMENTS}

The Natural Programming research has been performed in collaboration with over 30 students. The Natural Programming Project is funded in part by the National Science Foundation, under NSF grants IIS-0329090, IIS-0757511, CCF-0811610, and IIS-1116724, and as part of the EUSES consortium (End Users Shaping Effective Software) under NSF grant ITR CCR0324770. It has also been supported by SAP, Inc., Adobe, Inc., Microsoft Research, IBM, and the Korea Foundation for Advanced Studies (KFAS). Any opinions, findings and conclusions or recommendations expressed in this material are those of the author and do not necessarily reflect those of the National Science Foundation or any other funding source. 


\section{REFERENCES}

[1] B. A. Myers, J. F. Pane, and A. Ko, "Natural Programming Languages and Environments," Communications of the ACM, vol. 47, pp. 47-52, Sept 2004.

[2] A. J. Ko and B. A. Myers, "Debugging Reinvented: Asking and Answering Why and Why Not Questions about Program Behavior," in ICSE'2008: 30th International Conference on Software Engineering Leipzig, Germany, 2008, pp. 301-310.

[3] A. J. Ko and B. A. Myers, "Finding Causes of Program Output with the Java Whyline," in CHI'2009: Human Factors in Computing Systems Boston, MA, 2009, pp. 1569-1578.

[4] T. D. LaToza and B. Myers, "Developers Ask Reachability Questions," in ICSE'2010: Proceedings of the International Conference on Software Engineering Capetown, South Africa, 2010, pp. 185-194.

[5] T. D. LaToza and B. A. Myers, "Hard to Answer Questions about Code," in Second Workshop on Evaluation and Usability of Programming Languages and Tools (PLATEAU'2010) at SPLASH/Onward! 2010 Reno, NV, 2010.

[6] T. D. LaToza and B. A. Myers, "Visualizing Call Graphs," in VL/HCC'2011: IEEE Symposium on Visual Languages and Human-Centric Computing Pittsburgh, PA, 2011, pp. 117-124.

[7] B. Ellis, J. Stylos, and B. Myers, "The Factory Pattern in API Design: A Usability Evaluation," in International Conference on Software Engineering (ICSE'2007), Minneapolis, MN, 2007, pp. 302-312.

[8] J. Beaton, S. Y. Jeong, Y. Xie, J. Stylos, and B. A. Myers, "Usability Challenges for Enterprise ServiceOriented Architecture APIs," in 2008 IEEE Symposium on Visual Languages and Human-Centric Computing, $V L / H C C$ '08, Herrsching am Ammersee, Germany, 2008, pp. 193-196.
[9] J. Beaton, B. A. Myers, J. Stylos, S. Y. Jeong, and Y. Xie, "Usability Evaluation for Enterprise SOA APIs," in 2nd International Workshop on Systems Development in SOA Environments, SDSOA 2008 (Co-located with ICSE 2008), Leipzig, Germany, 2008, pp. 29-34.

[10] J. Stylos and S. Clarke, "Usability Implications of Requiring Parameters in Objects' Constructors," in International Conference on Software Engineering (ICSE'2007), Minneapolis, MN, 2007, pp. 529-539.

[11] J. Stylos and B. A. Myers, "Mica: A Programming WebSearch Aid," in IEEE Symposium on Visual Languages and Human-Centric Computing, VL/HCC'06, Brighton, UK, 2006, pp. 195-202.

[12] J. Stylos, A. Faulring, Z. Yang, and B. A. Myers, "Improving API Documentation Using API Usage Information," in VL/HCC'09: IEEE Symposium on Visual Languages and Human-Centric Computing Corvallis, Oregon, 2009, pp. 119-126.

[13] S. Y. Jeong, Y. Xie, J. Beaton, B. A. Myers, J. Stylos, R. Ehret, J. Karstens, A. Efeoglu, and D. K. Busse, "Improving Documentation for eSOA APIs Through User Studies," in Second International Symposium on End User Development (IS-EUD'2009), Siegen, Germany, 2009, pp. 86-105.

[14] D. S. Eisenberg, J. Stylos, A. Faulring, and B. A. Myers, "Using Association Metrics to Help Users Navigate API Documentation," in 2010 IEEE Symposium on Visual Languages and Human-Centric Computing (VL/HCC'10) Leganés-Madrid, Spain, 2010, pp. 23-30.

[15] M. Mooty, A. Faulring, J. Stylos, and B. A. Myers, "Calcite: Completing Code Completion for Constructors using Crowds," in 2010 IEEE Symposium on Visual Languages and Human-Centric Computing (VL/HCC'10) Leganés-Madrid, Spain, 2010, pp. 15-22. 